\title{
Conhecimento dos cirurgiões-dentistas sobre Banco de Dentes Humanos
}

\author{
Daylana Pacheco da Silva*; Urias Silva Vasconcelos*; Adelmar Pereira da Silva Neto**; Gregório \\ Antônio Soares Martins***; Graciela Maria Oliveira Sipaúba****; Walter Leal de Moura*****; \\ Carmem Dolores Vilarinho Soares de Moura*****
}

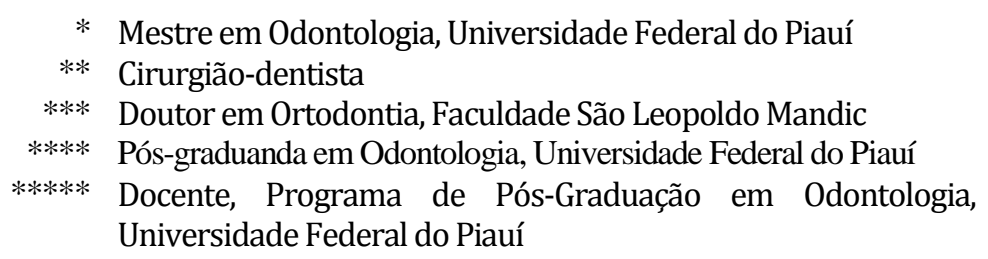

Recebido em 05/04/2017. Aprovado em 01/06/2017.

\begin{abstract}
RESUMO
Banco de dentes humanos (BDH) é uma entidade sem fins lucrativos que tem a finalidade de recepcionar, armazenar, catalogar e manter em boa conservação dentes humanos para pesquisa e treinamento laboratorial pré-clínico de estudantes. No entanto, muitos cirurgiões-dentistas desconhecem a importância do BDH. O objetivo deste estudo foi avaliar o conhecimento dos cirurgiões-dentistas das Unidades Básicas de Saúde (UBS) do município de Teresina/PI sobre condutas diversas relacionadas aos BDHs. O presente estudo é do tipo observacional com amostragem probabilística estratificada com alocação proporcional, que utilizou entrevistas. Mais da metade dos cirurgiões-dentistas afirmaram ter conhecimento da existência de BDHs no Brasil, porém 81\% desconheciam a existência do BDH da Universidade Federal do Piauí (UFPI). Todos os entrevistados foram a favor da doação e consideram o dente como um órgão, entretanto 95,2\% nunca fez doação de dentes para um BDH. O lixo foi o destino mais comum após exodontias. Diante disto, foi possível concluir que não há doação de dentes humanos pelos cirurgiões-dentistas entrevistados, possivelmente, devido ao desconhecimento da existência do BDH da UFPI e suas peculiaridades.
\end{abstract}

Descritores: Dente. Ética. Obtenção de Tecidos e Órgãos.

\section{INTRODUÇÃO}

Com o intuito de atender às necessidades de ensino e pesquisa, o Banco de Dentes Humanos (BDH) é uma entidade sem fins lucrativos, que armazena e mantém dentes extraídos em boa conservação, respeitando normas éticas e legais para doação deste órgãoํ․

Assim, a criação do BDH nos cursos de Odontologia legaliza o uso dos dentes para fins acadêmicos e de pesquisa, direcionando sua 
captação, seguindo normas de acordo com as leis vigentes no país².

No Brasil, a normatização do BDH segue a resolução $n^{0}$ 441/11 do Conselho Nacional de Saúde e na Lei de Transplante de 1997, que reconheceu os dentes como órgãos do corpo humano ${ }^{3-5}$. A legislação prevê penalidade de 3 a 8 anos de reclusão e multa, para quem remover postmortem, órgãos, tecidos e partes do corpo humano de pessoa não identificada ${ }^{4,5}$. No entanto, ainda não há uma lei que contemple este tema para fins de ensino e extensão, visto que a legislação supracitada se refere apenas à pesquisa ${ }^{2}$. Portanto, o BDH busca estimular a doação de dentes, bem como sua legalização, a fim de inibir o comércio ilegal $^{2,6}$.

O descumprimento de normas éticas torna a manipulação de dentes um risco potencial de infecção cruzada, uma vez que os tecidos periodontais e pulpares apresentam patógenos salivares e sanguíneos ${ }^{7}$. Desta forma, para que os dentes sejam empregados em pesquisas, treinamentos laboratoriais, calibragem de estudos epidemiológicos e ensino ${ }^{6,7}$, é recomendado o uso de equipamentos de proteção individual (EPI), vacinação contra hepatite $B$ e protocolos de esterilização/desinfecção de dentes humanos ${ }^{7}$. No entanto, a escolha do método de esterilização dos dentes extraídos pode provocar alterações estruturais no esmalte e dentina ${ }^{8}$. Devido à inexistência de um método ideal, estudos buscam resultados favoráveis de materiais que agreguem a eficácia antimicrobiana à preservação estrutural do substrato dental.

Desta forma, o estudo visou avaliar o conhecimento dos cirurgiões-dentistas das Unidades Básicas de Saúde (UBS) do município de Teresina/PI sobre condutas diversas relacionadas ao BDH. A partir dos dados obtidos, será possível traçar estratégias para o incentivo à doação de dentes, bem como a conscientização dos profissionais sobre a importância do $\mathrm{BDH}$ para a formação acadêmica e a pesquisa.

\section{METODOLOGIA}

Este estudo foi aprovado pelo Comitê de Ética em Pesquisa da Universidade Federal do Piauí (UFPI), parecer $n^{0}$ 1.872.436. Os participantes foram informados sobre os objetivos da pesquisa e assinaram um termo de consentimento livre e esclarecido (TCLE), obedecendo a Resolução do Conselho Nacional de Saúde CNS n 466/12.

Trata-se de um estudo observacional transversal, conduzido com cirurgiões-dentistas inseridos na Estratégia de Saúde da Família alocados nas UBS do município de Teresina/PI. Um questionário foi elaborado a partir de estudo anterior $^{7}$ e aplicado por meio de uma entrevista por um acadêmico previamente treinado em estudo-piloto. Coletaram-se informações sobre conhecimento, objetivos, aspectos éticos, legais e de biossegurança relacionados ao BDHs.

A amostra foi estabelecida por amostragem estratificada com alocação proporcional às regionais de saúde (quadro 1). Para este cálculo amostral, foi adotado o intervalo de confiança de $95 \%{ }^{9}$, utilizando dados referentes à taxa de resposta ( $\operatorname{sim}=94 \%$ ou não=6\%) do questionário aplicado por Pinto et al., 20097. As UBS foram sorteadas até atingir o número amostral necessário para cada regional.

Quadro 1. Distribuição de cirurgiões-dentistas em cada regional de saúde

\begin{tabular}{|ccc|}
\hline $\begin{array}{c}\text { Localização da } \\
\text { Regional }\end{array}$ & $\begin{array}{c}\text { População de } \\
\text { cirurgiões- } \\
\text { dentistas }\end{array}$ & $\begin{array}{c}\text { Amostra } \\
\text { (n) }\end{array}$ \\
Sul & 71 & 20 \\
Leste/Sudeste & 80 & 22 \\
Norte & 76 & 21 \\
Total & 227 & 63 \\
\hline
\end{tabular}


Os dados foram analisados no programa Statistical Package for the Social Science (SPSS ${ }^{\circledR}$, versão 20.0, para Windows, SPSS Inc., Chicago, IL, USA). Realizou-se análise descritiva das variáveis investigadas com valores absolutos e porcentagens.

\section{RESULTADOS}

Dos participantes deste estudo, 60,3\% $(n=38)$ eram do gênero feminino e 39,7\% (n=25) masculino.

De acordo com a tabela 1 , mais da metade (66\%) dos cirurgiões-dentistas tem conhecimento da existência do BDH no Brasil, contudo 81\% desconhecem o BDH da UFPI. Todos os entrevistados reconhecem o risco de infecção cruzada durante a manipulação de dentes humanos, e mesmo assim 23,8\% manipularam dentes sem o uso de EPI.

No que se refere à doação de dentes para um BDH, 95,2\% dos entrevistados nunca o fez. Entretanto, 66,7\% afirmaram que já lhe foram solicitados dentes para uso laboratorial ou pesquisa. De acordo com o estudo, 90,5\% doariam dentes próprios ao BDH (tabela 1).

Ao serem indagados sobre 0 armazenamento e a conduta de desinfecção, $34,9 \%$ armazenam e destes apenas 31,7\% realizam a desinfecção (tabela 2).

Com relação à conduta sobre o destino final dado aos dentes após exodontias, foram obtidas variadas respostas. A maioria afirmou descartar os dentes humanos no lixo ou entregar aos pacientes (gráfico 1).

Tabela 1. Conhecimento dos cirurgiões-dentistas das Unidades Básicas de Saúde de Teresina/PI sobre aspectos éticos, legais e de biossegurança relacionados ao $\mathrm{BDH}$.

\begin{tabular}{lcc}
\multicolumn{1}{c}{ Questões } & Sim (\%) & Não (\%) \\
\hline Você tem conhecimento da existência de BDHs? & $42(66 \%)$ & $21(33 \%)$ \\
Você sabia que a UFPI tem BDH? & $12(19 \%)$ & $51(81 \%)$ \\
Já fez doação de dentes para BDH? & $3(4,8 \%)$ & $60(95,2 \%)$ \\
É a favor da doação de órgãos? & $63(100 \%)$ & - \\
Considera o dente um órgão? & $63(100 \%)$ & - \\
$\begin{array}{l}\text { Informa aos pacientes que o dente é um órgão e como tal segue } \\
\text { a lei de Transplante? }\end{array}$ & $14(22,2 \%)$ & $49(77,8 \%)$ \\
Você já manipulou dentes sem utilizar EPI? & $15(23,8 \%)$ & $48(76,8 \%)$ \\
Sabia que na polpa e nos tecidos periodontais podem existir & $63(100 \%)$ & - \\
patógenos sanguíneos transmissíveis ao profissional? & & \\
Já lhe solicitaram dentes para uso em laboratórios e pesquisa? & $42(66,7 \%)$ & $21(33,3 \%)$ \\
Doaria dentes próprios para BDH? & $57(90,5 \%)$ & $6(9,5 \%)$ \\
\hline
\end{tabular}


Tabela 2. Desinfecção de dentes realizada por cirurgiões-dentistas das Unidades Básicas de Saúde em Teresina/PI

\begin{tabular}{lcc}
\multicolumn{1}{c}{ Questões } & Sim & Não \\
\hline Você armazena dentes? & $34,9 \%$ & $65,1 \%$ \\
Se sim, você realiza desinfecção antes de armazená-los? & $31,7 \%$ & $3,2 \%$ \\
\hline
\end{tabular}

Gráfico 1. Conduta dos cirurgiões-dentistas de Teresina/PI sobre o destino final dado aos dentes após exodontias nas Unidades Básicas de Saúde

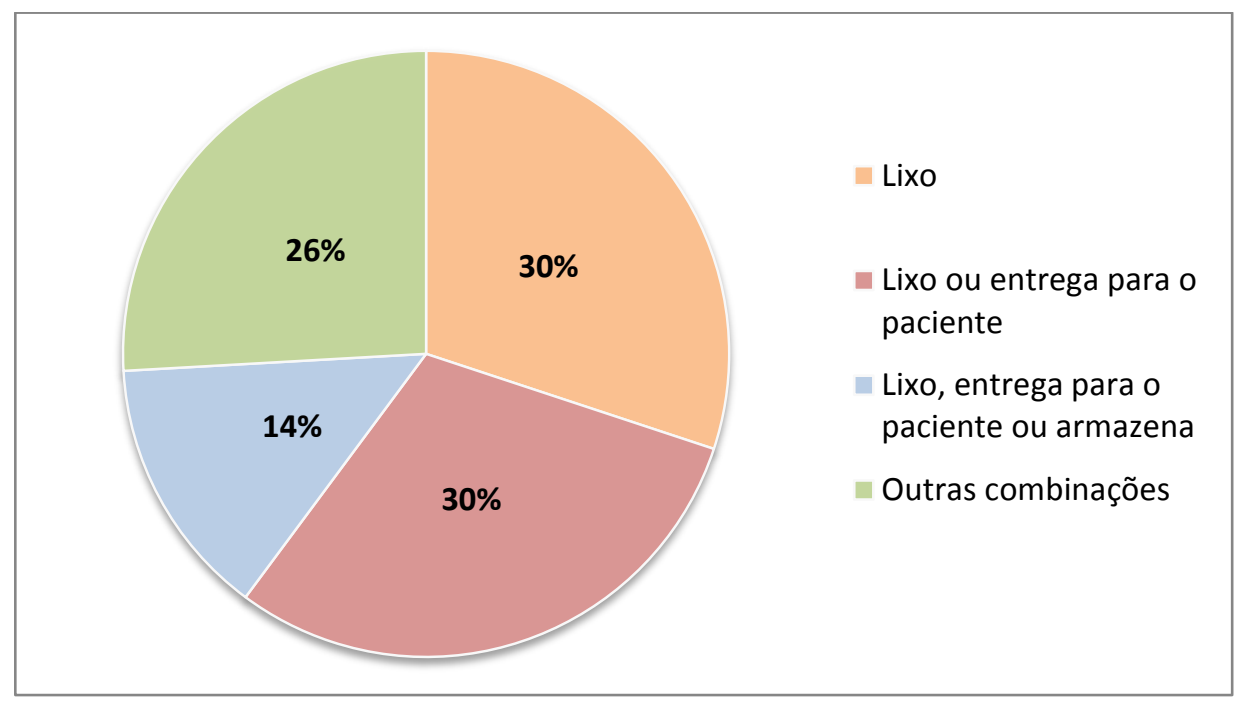

\section{DISCUSSÃO}

A presença de BDH nos cursos de Odontologia ocorre pela necessidade de gerenciamento biológico, armazenamento ou descarte dos dentes extraídos utilizados em disciplinas e pesquisas dos alunos da Graduação ${ }^{10}$, contemplando a importância do elemento dental como órgão funcional e integrante do sistema estomatognático ${ }^{11}$.

No presente estudo, a maioria dos profissionais relatou conhecer a existência do $\mathrm{BDH}$ no Brasil, no entanto desconheciam o BDH da UFPI, bem como suas atividades e condutas. $\mathrm{O}$ mesmo foi relatado no estudo de Pinto et al., $2009^{12}$. Estudos prévios atribuem este fato à vinculação recente do $\mathrm{BDH}$ às faculdades de Odontologia $^{12-14}$.
Todos os entrevistados relataram ser a favor da doação de órgãos, além de considerar o dente como órgão, corroborando com o estudo de Pinto et al., 2009². Entretanto, a informação dada aos pacientes sobre a lei de Transplante Brasileira foi negligenciada. Assim, é notório que muitos dos profissionais questionados cometeram falhas éticas e legais com relação ao destino dos dentes extraídos.

Ao serem indagados sobre a requisição de dentes para fins científicos e laboratoriais, os entrevistados afirmaram terem sido abordados para tal. Para Freitas et al. ${ }^{14}$ este órgão ainda é pouco valorizado pelos pesquisadores e uma grande quantidade de dentes é empregada em estudos laboratoriais, ferindo princípios éticos e legais. 
O comércio ilegal de dentes humanos extraídos é considerado crime ${ }^{15}$. O artigo $15^{\circ}$ do Código Penal prevê pena de 3 a 8 anos de reclusão para quem comprar ou vender tecidos, órgãos ou partes do corpo humano ${ }^{4,5}$. De acordo como Código de Ética Odontológica, o descumprimento das legislações que regulam o uso de órgão é considerado infração ética, podendo determinar penalidades que vão desde advertência à cassação do exercício da profissão.

Com relação ao conhecimento e condutas de biossegurança, todos os entrevistados afirmaram conhecer o risco de contaminação, através de patógenos sanguíneos transmissíveis, contudo negligenciam o uso de EPI durante a manipulação dos dentes. Para Moreira et al. ${ }^{13} \mathrm{e}$ Dominici et al. ${ }^{6}$, o uso de EPI e a desinfecção de dentes diminuem o risco de infecções cruzadas, ressaltando a importância do BDH. Por outro lado, o modo de armazenamento e de desinfecção de dentes pode influenciar nas propriedades de seus tecidos. Materiais como formol, hipoclorito de sódio, etanol e glutaraldeído podem alterar a permeabilidade e a resistência adesiva da dentina $^{16,17}$. O protocolo de armazenamento recomendado pelo Banco de Dentes da UFPI é o uso de água destilada em frasco fechado refrigerado, trocada semanalmente. A técnica de esterilização será aplicada conforme o objetivo da sua utilização pelo aluno ou pesquisador, sendo a autoclave o método mais aplicado.

Sobre a conduta dada aos dentes após exodontia, a maioria dos entrevistados afirmou descartar no lixo ou entregar para o paciente. Tais atitudes podem ser explicadas pela falta de conhecimento sobre a existência e atividades do $\mathrm{BDH}$ na UFPI, como também das Resoluções ANVISA 306/2004 ${ }^{18}$ e CONAMA 358/2005 que versam sobre aspectos técnicos que regulamentam o gerenciamento de Resíduos de Serviços de Saúde (RSS), contrariando a atitude de entregar o dente extraído ao paciente.
Na elaboração do questionário o item descarte no lixo especificou condutas de manejo: segregação, acondicionamento e destino final. A Resolução ANVISA 306/2004 considera que os serviços de saúde são os responsáveis pelo correto gerenciamento de todos os RSS por eles gerados, atendendo as normas e exigências legais, desde o momento de sua geração até o seu destino final. Todo gerador deve elaborar um Plano de Gerenciamento de Resíduos de Serviços de Saúde (PGRSS), baseado nas características dos resíduos gerados e na classificação constante na resolução, estabelecendo as diretrizes de manejo.

\section{A Resolução CONAMA 358/2005} também dispõe sobre o tratamento e disposição final dos RSS e outras providências, classificandoos em Grupos. Os resíduos odontológicos incluídos no Grupo A4 (órgãos ou tecidos provenientes de procedimentos cirúrgicos ou de estudos anatomopatológico ou de confirmação diagnóstica), devem ser encaminhados, após acondicionados nas devidas embalagens, sem tratamento prévio para local devidamente licenciado para destino final dos RSS. O não cumprimento do disposto nesta Resolução, fiscalizada pelo Sistema Nacional de MeioAmbiente (SISNAMA), sujeitará o infrator as penalidades e sansões previstas na legislação, em especial, na lei 9.605 de 12 de fevereiro de 1998 e no seu decreto regulamentado.

O Banco de Dentes da UFI foi criado em setembro de 2006, pela Resolução No 151/06 do Conselho de Ensino, Pesquisa e Extensão, Processo N 23111.010340/04-11. A não doação de dentes extraídos ao Banco de Dentes da UFI pode ser explicada pelo desconhecimento de sua existência, cabendo a esta instituição elaborar documento informando esta Resolução e os procedimentos para doação. O ideal para casos de extração de um ou mais dentes é obter a autorização do paciente para a doação e informálo sobre a finalidade e objetivos do BDH. Após 
sua aceitação, deve-se preencher um TCLE explicitando todo o trâmite para doação ${ }^{18}$. A função de um BDH é facilitar a doação, preocupando-se com a procedência e destino para tal. $^{20}$

Com relação a possibilidade de doação de algum dente próprio, os cirurgiões-dentistas responderam positivamente, o que corroborou com estudo anterior ${ }^{12}$. Vale ressaltar que no presente estudo, não foi considerado tempo ou grau de formação (clínico geral ou especialista) dos profissionais.

Apesar das limitações, espera-se que este estudo possa resultar em atitudes de esclarecimento aos profissionais e pacientes, contribuindo assim para melhorar a qualidade do conhecimento sobre doação aos BDH, e manejo dos resíduos dos serviços de saúde. A doação oficial de dentes possibilita novas pesquisas e combate o comércio ilegal de dentes no meio acadêmico. Assim, propõese uma reciclagem específica por meio de palestras esclarecedoras e outras atividades pelo gestor municipal aos CDs da rede (ESF), inserindo a Universidade e o CRO do Estado nestas ações.

\section{CONCLUSÕES}

Conclui-se que não há doação de dentes humanos pelos cirurgiões-dentistas das UBS de Teresina/PI, possivelmente devido ao desconhecimento sobre o BDH da UFPI, sendo o lixo o principal destino final dos dentes.

\section{AGRADECIMENTOS}

Aos cirurgiões-dentistas das Unidades Básicas de Saúde do município de Teresina/PI que colaboraram com a execução desta pesquisa.

\section{ABSTRACT \\ Knowledge of dental surgeons about Human Teeth Bank}

Human teeth bank (BDH) is a non-profit organization whose purpose is to receive, store, catalog and maintain human teeth for research and pre-clinical laboratory training of students. However, many dental surgeons are unaware of the importance of $\mathrm{BDH}$. The objective of this study was to evaluate the knowledge of dental surgeons of the Basic Health Units of the city of Teresina/PI about their behaviors related to BDHs. We performed an observational study with probabilistic sampling stratified with proportional allocation, which used interviews. More than half of the dental surgeons reported knowing of the existence of BDHs in Brazil, but $81 \%$ were unaware of the existence of the $\mathrm{BDH}$ of the Federal University of Piauí (UFPI). All interviewees were in favor of the donation and considered the tooth as an organ. However, 95.2\% never donated teeth to a BDH. Trash was the most common destination after exodontia. Given this, it was possible to conclude that there is no donation of human teeth by the dentists interviewed, possibly due to the lack of knowledge of the existence of the UFPI's BDH and its peculiarities.

Descriptors: Tooth. Ethics. Tissue and Organ Procurement.

\section{REFERÊNCIAS}

1. Motta-Murguia L, Saruwatari-Zavala G. Mexican regulation of Biobanks. J Law Med Ethics. 2016 Mar;44(1):58-67.

2. Quinto PD. Banco de dentes humanos no Brasil: revisão de literatura. Rev ABENO. 2012;12(2):178-84.

3. Nassif ACS, Tieri F, Ana PA, Botta SB, Imparato JCP. Estrutura de um Banco de Dentes Humanos. Pesqui Odontol Bras. 2003; 17(Supl1):70-4.

4. Conselho Nacional de Saúde. Resolução $n^{0}$ 441, de 12 de maio de 2011. Diretrizes para análise ética de projetos de pesquisas que envolvam armazenamento de material biológico humano ou uso de material armazenado em pesquisas anteriores. Diário Oficial União 18 de jul 2011; Seção 1.

5. Conselho Nacional de Saúde. Resolução 466, 
de 12 de dezembro de 2012. Aprova as diretrizes e normas regulamentadoras de pesquisa envolvendo seres humanos. Diário Oficial da União 13 jun 2013; Seção 1.

6. Dominici JT, Eleazer PD, Clark SJ, Staat RH, Scheetz JP. Disinfection/sterilization of extracted teeth for dental student use. J Dent Educ. 2001;65(11):1278-80.

7. DeWald JP. The use of extracted teeth for in vitro bonding studies: a review of infection control considerations. Dent Mater. 1997;13(2):74-81.

8. Silva MF, Mandarino F, Sassi JF, Menezes M, Centola ALB, Nonaka T. Influência do tipo de armazenamento e do método de desinfecção de dentes extraídos sobre a adesão à estrutura dental. Rev Odont Univ Cid São Paulo. 2006;18(2):175-80.

9. Bolfarine B. Elementos de Amostragem. São Paulo: Blucher; 2005.

10. Imparato, JCP. Banco de dentes humanos. Ed. FOUSP; 2003.

11. Júnior ECS, Guimarães CC, Marques AAF, Rebelo MAB, Conde NCO, Bandeira MFCL, Pereira JV. Banco de dentes humanos e educação em saúde na Universidade Federal do Amazonas. Relato de experiência. Rev ABENO. 2012; 12 (2):185-9.

12. Pinto S.L, Silva SP, Barros LM, Tavares EP, Silva JBOR, Freitas ABDA. Conhecimento popular, acadêmico e profissional sobre o banco de dentes humanos. Pesqui Bras Odontopediatria Clín Integr. 2009; 9(1):101-6.

13. Moreira L, Genari B, Stello R, Collares FM, Samuel SMW. Banco de dentes humanos para o ensino e pesquisa em Odontologia. Rev Fac Odontol Porto Alegre. 2009; 50(1):34-37.

14. Freitas ABDA, Castro CDL, Sett GS, Barros LM, Moreira A N, Magalhães CS. Uso de dentes extraídos nas pesquisas odontológicas publicadas em periódicos Brasileiros de acesso online gratuito: um estudo sob o prisma da bioética. Arqu Odontol. 2010; 46(3):136-43.

15. Silva ACC, França ECS, Carvalho RLS, Leão EC. Levantamento quantitativo dos dentes humanos solicitados no primeiro semestre do ano de 2001 nos cursos de odontologia do estado de Pernambuco. An Fac Odontol Univ Fed Pernamb 2001; 11(1/2):29-32.

16. Costa S, Mameluque S, Brandão EL, Melo ALMA, et al. Dentes humanos no ensino odontológico: procedência, utilização, descontaminação e armazenamento pelos acadêmicos da Unimontes. Rev ABENO. 2005; 7(1):6-12.

17. Poletto MM, Moreira M, Dias MM, Lopes MGK, Pizzato E, Lavoranti OJ. Banco de dentes humanos: perfil sócio-cultural de um grupo de doadores. Rev Gaúcha Odontol. 2010; 58(1):91-4.

18. Agência Nacional de Vigilância Sanitária. Resolução RDC n. 306, de 07 de dezembro de 2004. Dispõe sobre o Regulamento Técnico para o gerenciamento de resíduos de serviços de saúde. Diário Oficial da União. 2004; 10 dezembro.

19. Conselho Nacional de Meio Ambiente. Resolução n. 358, de 29 de abril de 2005. Dispõe sobre o tratamento e a disposição final dos resíduos dos serviços de saúde e dá outras providências. Diário Oficial da União 2005; 04 maio.

20. Gomes GM, Gomes G, Pupo YM, Gomes OMM, et al. Utilização de dentes humanos: aspectos éticos e legais. Rev Gaúcha Odontol. 2013; 61(1):477-83.

Correspondência para:

Daylana Pacheco da Silva

e-mail: daylanapachecos@gmail.com

Rua Santa Cruz 1213, Bairro Alto

13419-030 Piracicaba/SP 